Artigo Original

Original Article

\title{
Erro técnico de medida em antropometria: análise de precisão e exatidão em diferentes plicômetros
}

\section{Technical Error of Measurement in Anthropometry: Analysis of Precision and Accuracy in Different Plicometers}

Gustavo dos Santos Ribeiro ${ }^{1,4}$ MS; Émerson Barata Fragoso² Esp; Rodrigo D’Azevedo Nunes³ ${ }^{3}$ André Luiz Lopes $^{\S 4} \mathrm{PhD}$

Recebido em: 27 de abril de 2019. Aceito em: 05 de junho de 2019.

Publicado online em: 31 de julho de 2019.

\section{Resumo}

Introdução: Existem diferentes plicômetros destinados a mensurar dobras cutâneas. No entanto, pouco se sabe sobre a confiabilidade de suas medidas.

Objetivo: Avaliar a precisão e a exatidão de nove modelos utilizados para mensurar as dobras cutâneas.

Métodos: Foram selecionados por conveniência nove equipamentos: Body Caliper, Innovare, Lange, Slim Guide, Neo Prime, Harpenden, Holtain, Premier e Digital. Utilizou-se um dispositivo desenvolvido com células de carga para mensurar a pressão das áreas de contato nas amplitudes de 10, 15, 20, 25, 30, 35, 40, 45 e $50 \mathrm{~mm}$. Foram calculadas medidas de precisão (ETM-INTRA), exatidão (ETM-INTER) e concordância (Bland-Altman). Adotou-se as medidas do plicômetro Harpenden como padrão-ouro.

Resultados: Observou-se precisão adequada nos plicômetros Holtain $(0,35 \pm 0,21)$, Harpenden $(0,39 \pm 0,35)$, Digital $(0,49 \pm 0,39)$, Premier $(0,61 \pm 0,52)$, Neo Prime $(0,49 \pm 0,39)$, Slim Guide $(0,54 \pm$ $0,41)$, Lange $(0,75 \pm 0,74)$ e Innovare $(0,83 \pm 0,63)$. Somente modelo Body Caliper não foi preciso $(3,93 \pm 2,53)$. Em relação à exatidão, somente os modelos Premier $(3,61 \pm 0,85)$, Digital $(4,44 \pm 3,26)$ e Innovare $(4,79 \pm 3,89)$ exibiram níveis aceitáveis de exatidão. Os demais equipamentos não se mostraram exatos (Slim Guide 15,03 \pm

\begin{tabular}{|l|}
\hline Pontos-Chave Destaque \\
- Foram examinados os \\
plicômetros: Body Caliper, \\
Innovare, Lange, Slim Guide, \\
Neo Prime, Holtain, Premier e \\
Digital. As medidas do \\
plicômetro Harpenden foram \\
consideradas como padrão- \\
ouro. \\
- O modelo Body Caliper não se \\
mostrou preciso. \\
- Somente os modelos Premier, \\
Digital e Innovare exibiram \\
níveis aceitáveis de exatidão. \\
\hline
\end{tabular}
7,99; Holtain 17,05 \pm 4,30; Neo Prime 23,82 \pm 10,35; Lange 57,88 \pm 9,47; Body Caliper 143,68 $\pm 33,03$ ).

Conclusão: Os modelos Harpenden, Innovare, Premier e Digital são os plicômetros que se mostraram mais precisos e exatos para mensurar dobras cutâneas.

Palavras-chave: antropometria, composição corporal, pregas cutâneas, análise de falha de equipamento.

\begin{abstract}
Introduction: There are different calipers for measuring skinfolds. However, little is known about the reliability of its measures.
\end{abstract}

\footnotetext{
$\S$ Autor correspondente André Luiz Lopes - e-mail: andregym23@gmail.com Afiliações: ${ }^{1}$ Universidade Federal de Ciências da Saúde de Porto Alegre (UFCSPA), Porto Alegre, Brasil; ${ }^{2}$ Centro Universitário La Salle (LA SALLE), Canoas, Brasil; ${ }^{3}$ Faculdade SOGIPA de Educação Física, Porto Alegre, Brasil; ${ }^{4}$ Instituto ISulBra/Faculdades QI, Porto Alegre, Brasil.
} 
Objective: To evaluate the precision and accuracy of nine skinfold calipers.

Methods: Body Caliper, Innovare, Lange, Slim Guide, Neo Prime, Harpenden, Holtain, Premier, and Digital models were convenience selected. A device developed with load cells was used to measure jaw pressure at 10,15, 20, 25, 30, 35, 40, 45- and 50-mm. Accuracy measurements (TEM-INTRA), reproducibility (TEM-INTER), and agreement (Bland-Altman analysis) were calculated. It was adopted as measures of the Harpenden Caliper as gold standard.

Results: Adequate accuracy was observed in Holtain $(0.35 \pm 0.21)$, Harpenden (0.39 \pm 0.35$)$, Digital $(0.49 \pm 0.39)$, Premier $(0.61 \pm 0,52)$, Neo Prime ( $0.49 \pm 0.39)$, Slim Guide $(0.54 \pm 0.41)$, Lange $(0.75 \pm 0.74)$ and Innovare $(0.83 \pm 0.63)$ calipers. Only Body Caliper model was not accurate (3.93 \pm 2.53$)$. Regarding TEM-INTER, only the Premier (3.61 $\pm 0.85)$, Digital $(4.44 \pm 3.26)$ and Innovare $(4.79 \pm 3.89)$ models showed acceptable levels. The others equipment did not showed reproducibility (Slim Guide $15.03 \pm 7.99$; Holtain $17.05 \pm 4.30$; Neo Prime $23.82 \pm 10.35$; Lange (57.88 \pm 9.47; Body Caliper $143.68 \pm$ 33.03).

Conclusion: The Harpenden, Innovare, Premier and Digital models showed to be most accurate and exact skinfold caliper for measuring skinfolds.

Keywords: anthropometry, body composition, skinfold thickness, equipment failure analysis.

\section{Erro técnico de medida em antropometria: análise de precisão e exatidão em diferentes plicômetros}

\section{Introdução}

A composição corporal é uma ferramenta amplamente utilizada por profissionais da saúde e do esporte para acompanhar os efeitos de uma intervenção e/ou predizer índices de risco e desempenho esportivo(1). As dobras cutâneas (DC) são as principais medidas utilizadas nesta avaliação devido à sua alta correlação com densidade corporal, além do baixo custo operacional e boa confiabilidade(2,3). A variabilidade de dados pode ser reduzida ou conhecida ao calcularmos o erro técnico de medida (ETM) de cada avaliador. Esta técnica permite aos antropometristas verificarem sua precisão (ETM-INTRA) e sua exatidão (ETM-INTER), minimizando a possibilidade de viés na interpretação de dados $(1,4)$.

Um aspecto que vem sendo negligenciado ao longo dos anos refere-se à precisão, exatidão e a confiabilidade dos equipamentos usados para mensurar as DC e as medidas aferidas em modelos diferentes podem ser comparadas $(2,5,6)$. Existem poucas informações na literatura sobre o tema. Em 1955, Edwards et al.(7) mencionaram uma série de recomendações para auxiliar na construção destes equipamentos. A principal característica apontada pelos autores está relacionada com a abertura das hastes. Elas precisam exercer uma pressão constante (8-10 $\mathrm{g} / \mathrm{mm}^{2}$ ) ao longo de toda a amplitude, sendo que a haste superior do equipamento deve ser fixa e a inferior móvel. Além disso, as áreas de contato devem estar a $152,4 \mathrm{~mm}$ do eixo do dispositivo, denominado de pivô.

Com o crescimento do mercado fitness e wellness, as indústrias especializadas em materiais de avaliação aumentaram a produção e a venda destes equipamentos(8). No entanto, algumas premissas básicas parecem não terem sido aplicadas no desenvolvimento desses equipamentos. Atualmente, o mercado disponibiliza plicômetros em diversos formatos. Segundo Cyrino et al.(2), a mensuração das DC e a estimativa da massa adiposa podem ser afetadas pelo tipo de plicômetro utilizado, superfície de contato, distância e pressão das hastes, abertura das 
hastes e posicionamento das molas (Lei de Hooke).

Considerando estas informações, o objetivo do presente estudo foi avaliar a precisão e a exatidão de nove modelos de plicômetros comercializados dentro e fora do país, averiguando a confiabilidade de suas medidas para análise da composição corporal.

\section{Métodos}

No presente estudo transversal foram avaliados nove equipamentos para mensurar
DC. Os modelos clínicos Body Caliper (Valtro, Itália), Innovare (Cescorf, Brasil), Neo Prime (Prime Med, Brasil), Lange (Beta Tech, Estados Unidos) e Slim Guide (Rosscraft, Canadá) foram selecionados por conveniência, assim como os modelos científicos Harpenden (Baty Int, Inglaterra), Holtain (Crosswell, Inglaterra) e Premier (Cescorf, Brasil). Além destes, o modelo científico digital (Cescorf, Brasil) também foi selecionado para estudo. $\mathrm{O}$ Quadro 1 apresenta as especificações de cada equipamento.

\begin{tabular}{|l|c|c|c|c|}
\hline Modelo & Amplitude & Distância P-R & Molas & País de origem \\
\hline Cientifico & & & & \\
\hline Holtain & $46 \mathrm{~mm}$ & $117,0 \mathrm{~mm}$ & Indefinido $^{\mathrm{a}}$ & Inglaterra \\
\hline Harpenden & $80 \mathrm{~mm}$ & $152,4 \mathrm{~mm}$ & Oblíqua & Inglaterra \\
\hline Digital & $75 \mathrm{~mm}$ & $152,4 \mathrm{~mm}$ & Oblíqua & Brasil \\
\hline Premier & $85 \mathrm{~mm}$ & $152,4 \mathrm{~mm}$ & Oblíqua & Brasil \\
\hline Clínico & & & & \\
\hline Neo Prime & $60 \mathrm{~mm}$ & $230,0 \mathrm{~mm}$ & Sem molas & Brasil \\
\hline Slim Guide & $85 \mathrm{~mm}$ & $110,0 \mathrm{~mm}$ & Vertical & Canadá \\
\hline Lange & $60 \mathrm{~mm}$ & $75,0 \mathrm{~mm}$ & Vertical & Estados Unidos \\
\hline Innovare & $80 \mathrm{~mm}$ & $108,0 \mathrm{~mm}$ & Oblíqua & Brasil \\
\hline Body Caliper & $60 \mathrm{~mm}$ & $62,0 \mathrm{~mm}$ & Circular & Itália \\
\hline
\end{tabular}

P-R: distância entre o pivô e as áreas de contato.

${ }^{a}$ as molas situam-se na parte interna do equipamento, impossibilitando a sua visualização.

${ }^{\mathrm{b}}$ utiliza uma placa curva de alumínio em substituição ao tradicional sistema de molas.

\section{Procedimento experimental}

Todos os equipamentos foram calibrados de acordo com o fabricante. Utilizou-se um dispositivo desenvolvido com células de carga para mensurar a pressão exercida pelas molas de cada equipamento (áreas de contato) em diferentes amplitudes que foram escolhidas arbitrariamente $(10,15,20,25,30,35,40,45 \mathrm{e}$ $50 \mathrm{~mm}$ ). Foram realizadas duas medidas em cada amplitude, usando-se a média nos cálculos subsequentes. Uma terceira medida era realizada se os valores exibissem diferença superior a $2 \%$, com o valor discrepante sendo descartado.

\section{Análise de precisão, exatidão e confiabilidade}

As medidas de cada amplitude foram tabuladas no software Anthropotech 2.0 (ISULBRA, Brasil). O ETM foi calculado para cada amplitude fornecendo a medida de precisão do equipamento (ETM-INTRA).
Procedimento similar foi adotado para calcular a exatidão do modelo (ETM-INTER). Neste caso, utilizaram-se os valores do plicômetro científico Harpenden como padrão ouro devido a suas características respeitarem as normas propostas por Edwards et al.(7) . A rotina matemática utilizada pelo software para calcular estes dois parâmetros é descrita por Perini et al.(1) e Lopes \& Ribeiro(4). Adicionalmente, utilizou-se a análise de Bland-Altman para avaliar a confiabilidade das medidas de cada plicômetros(9).

\section{Análise estatística}

Os dados foram sumarizados em planilha eletrônica (Excel for Windows, Office 2010, USA) e analisados qualitativamente (ETMINTRA e ETM-INTER). O coeficiente de variação (CV) foi aplicado para determinar a heterogeneidade das medidas. O software GraphPAD Prism 5 (San Diego, USA) foi utilizado para análise descritiva e inferencial 
(Kruskal-Wallis), sendo significativo $\mathrm{p}<0,05$. A análise de Bland-Altman foi realizada no MedCalc Statistical Software version 19 (MedCalc Software, Ostend, Belgium). Os dados são expressos em média \pm desviopadrão.

\section{Resultados}

A Gráfico 1 apresenta o comportamento da pressão gerada pela abertura das hastes em cada amplitude investigada. Contatou-se diferença entre os modelos Harpenden vs Body

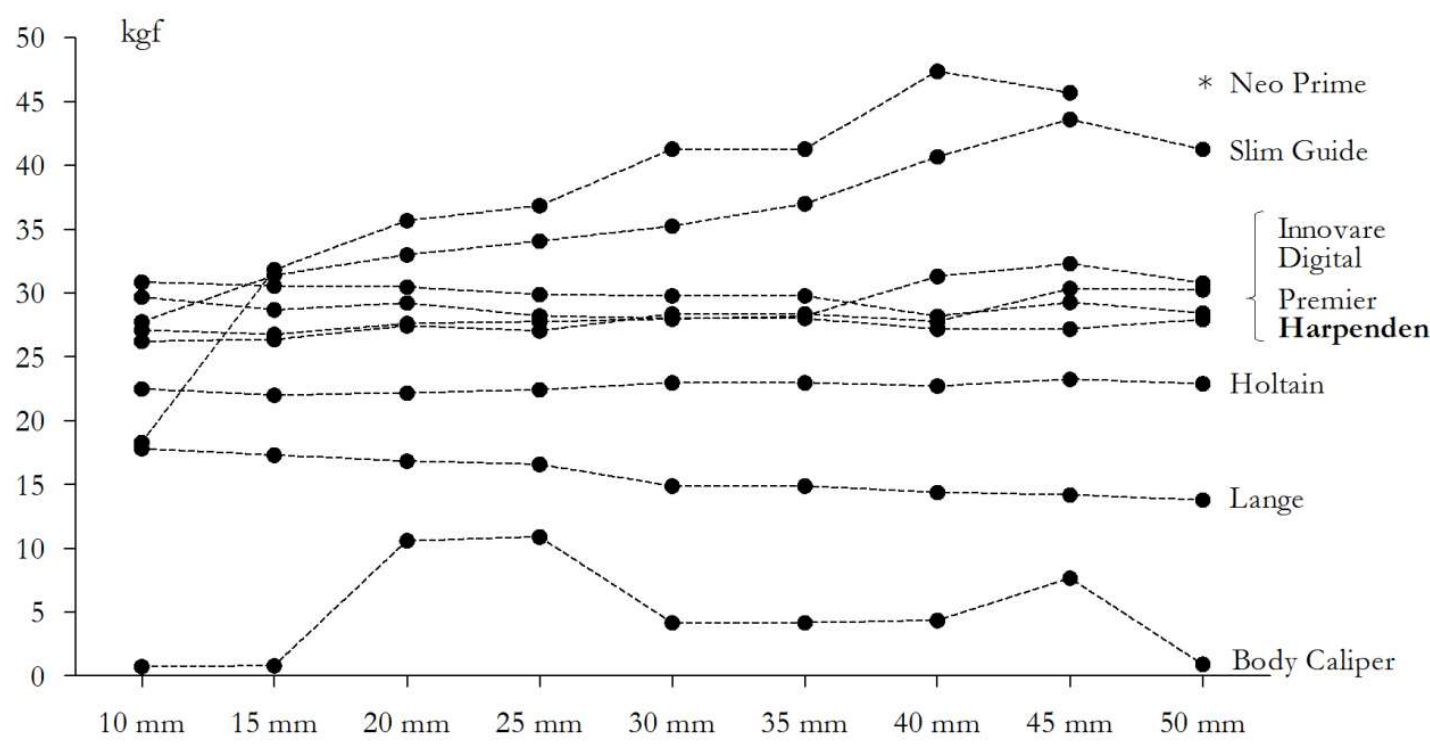

Gráfico 1 - Pressão das áreas de contato em diferentes aberturas. *Pressão superior a 50 kgf (limítrofe de aferição da célula de carga).

Body Caliper $(p<0.01)$, Premier vs Body Caliper e Lange $(\mathrm{p}<0.01)$, Digital vs Body Caliper $(\mathrm{p}<0.01)$, Innovare vs Body Caliper e Lange ( $<<0.05)$, Slim Guide vs Holtain, Lange e Body Caliper $(\mathrm{p}<0.05)$, Holtain vs Neo Prime $(p<0.05)$ e Neo Prime vs Lange e Body Caliper $(\mathrm{p}<0.05)$.

A Tabela 1 apresenta a análise de precisão e exatidão dos equipamentos. Todos os modelos exibiram precisão satisfatória, exceto o modelo Body Caliper que excedeu a limítrofe considerada adequada de $1 \%$. Todas as aferições se mostraram homogêneas em relação às amplitudes $(\mathrm{CV}<8 \%) \mathrm{e}$ equipamentos $(\mathrm{CV}<5 \%)$. Os modelos Harpenden e Premier obtiveram o melhor desempenho $(\mathrm{CV}<1 \%)$ enquanto o Body Caliper exibiu o pior resultado $(\mathrm{CV}=4,5 \%)$. Em relação à análise de exatidão, somente os modelos clínico, científico premier e científico digital da Cescorf demonstraram índices aceitáveis (ETM-INTER $<5 \%$ ). Os modelos Body Caliper e Lange obtiveram os piores valores de exatidão (ETM-INTER $>50 \%$ ).
Tabela 1 - Análise de precisão e exatidão dos equipamentos

\begin{tabular}{lcc}
\hline Modelo & $\begin{array}{c}\text { ETM-INTRA } \\
(\%)\end{array}$ & $\begin{array}{c}\text { ETM-INTER } \\
(\%)\end{array}$ \\
\hline \hline Científico & & \\
Holtain & $0,35 \pm 0,21$ & $17,05 \pm 4,30$ \\
Harpenden & $0,39 \pm 0,35$ & a \\
Digital & $0,49 \pm 0,39$ & $4,44 \pm 3,26$ \\
Premier & $0,61 \pm 0,52$ & $3,61 \pm 0,85$ \\
Clínico & & \\
Neo Prime & $0,49 \pm 0,39$ & $23,82 \pm 10,35$ \\
Slim Guide & $0,54 \pm 0,41$ & $15,03 \pm 7,99$ \\
Lange & $0,75 \pm 0,74$ & $57,88 \pm 9,47$ \\
Innovare & $0,83 \pm 0,63$ & $4,79 \pm 3,89$ \\
Body Caliper & $3,93 \pm 2,53$ & $143,68 \pm 33,03$ \\
\hline \multicolumn{2}{c}{ ETM: erro técnico de medida } \\
a Padrão ouro &
\end{tabular}

A Figura 1 apresenta a análise de BlandAltman dos diferentes plicômetros frente ao padrão-ouro. Observa-se que apenas os modelos Premier (A), Digital (B) e Innovare (C) apresentam níveis adequados de exatidão ( $\pm 5 \mathrm{~mm}$ ). Os modelos Holtain (D) e Lange (E) mostraram-se precisos, porém pouco exatos. 
A

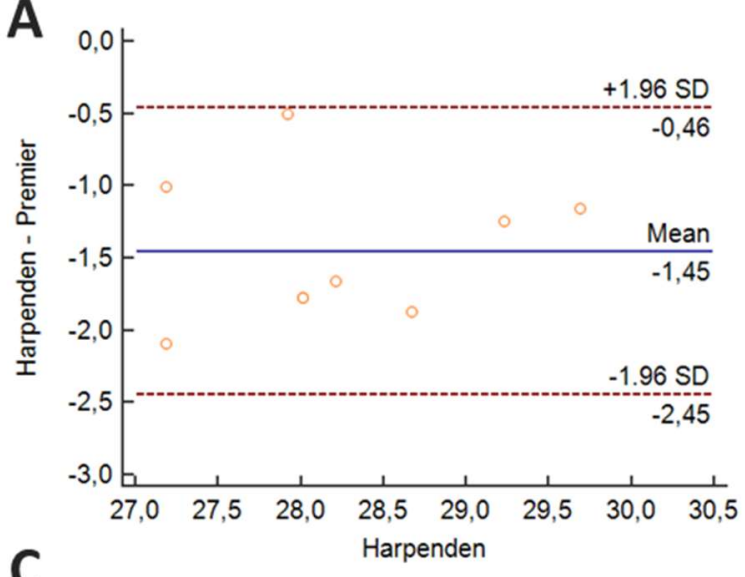

C

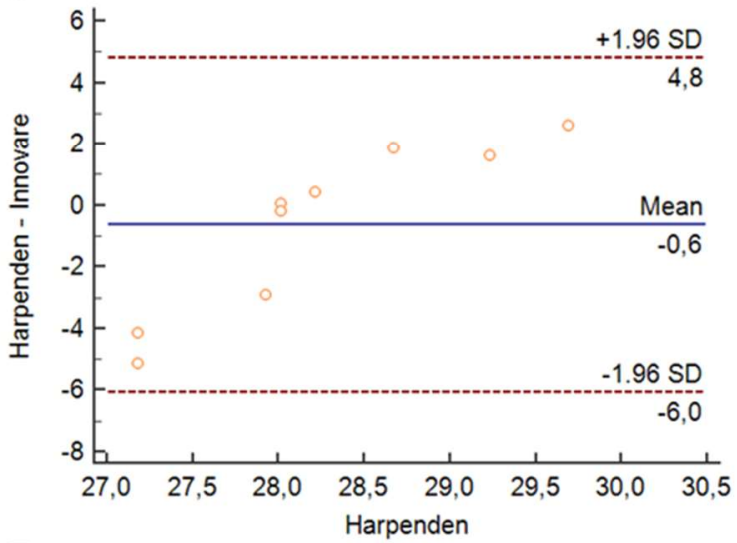

E

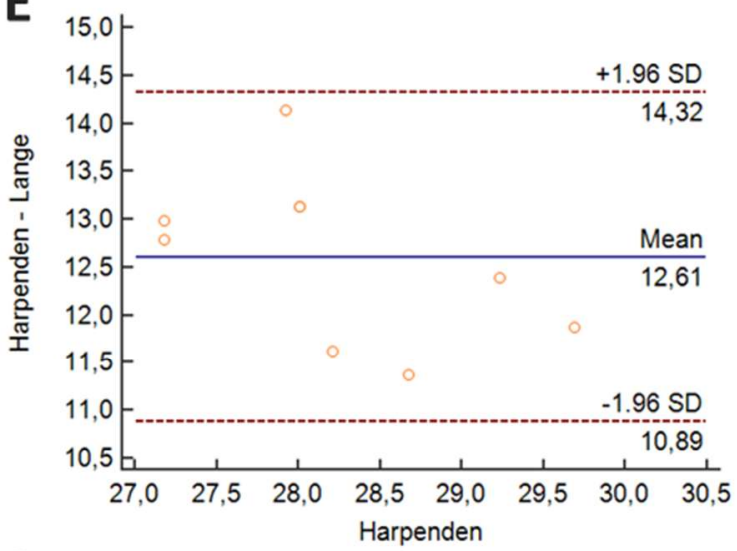

G

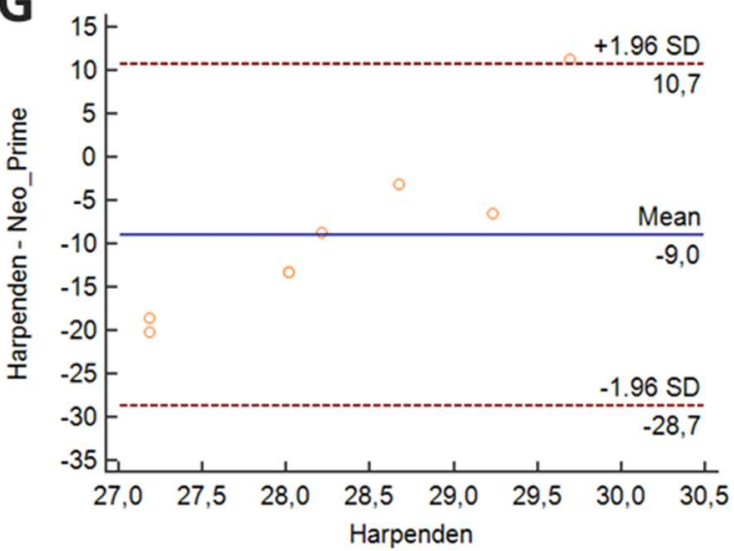

B

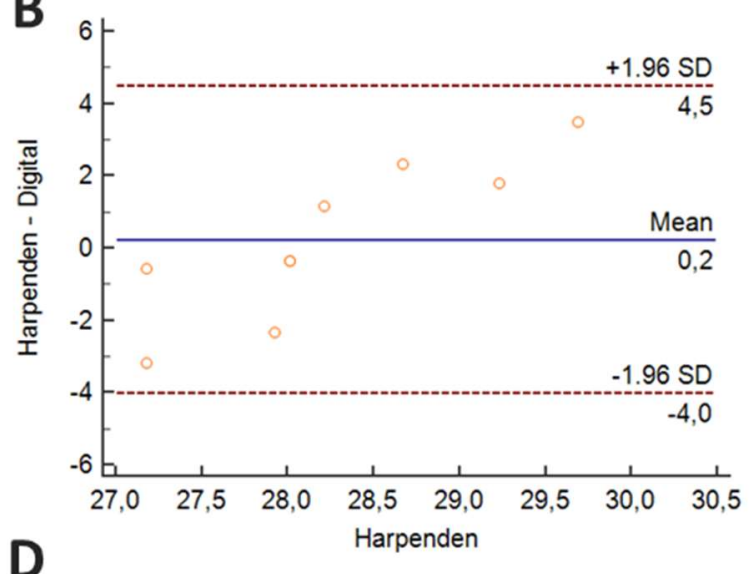

D

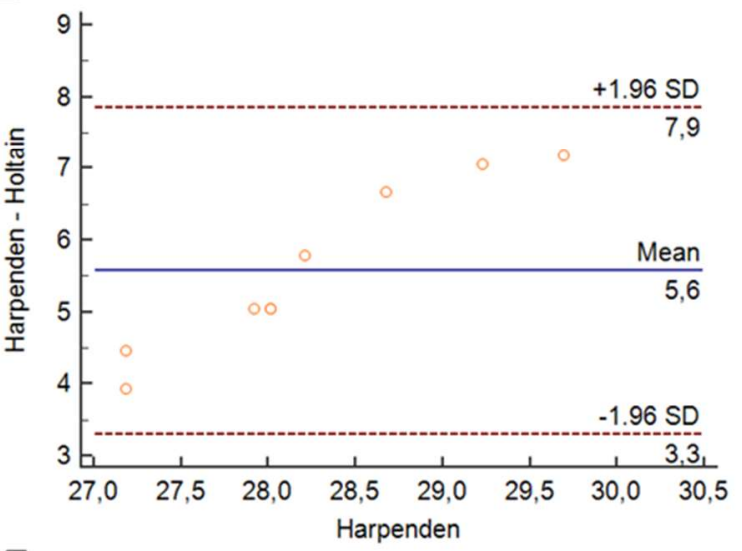

$\mathbf{F}$

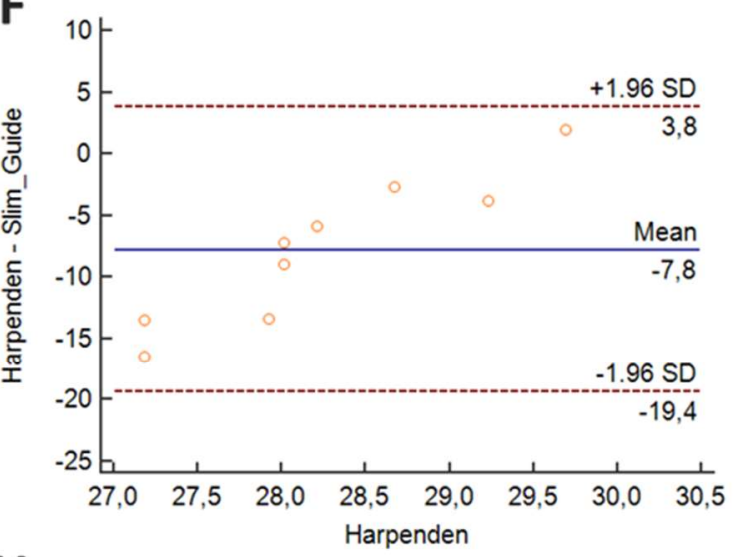

H

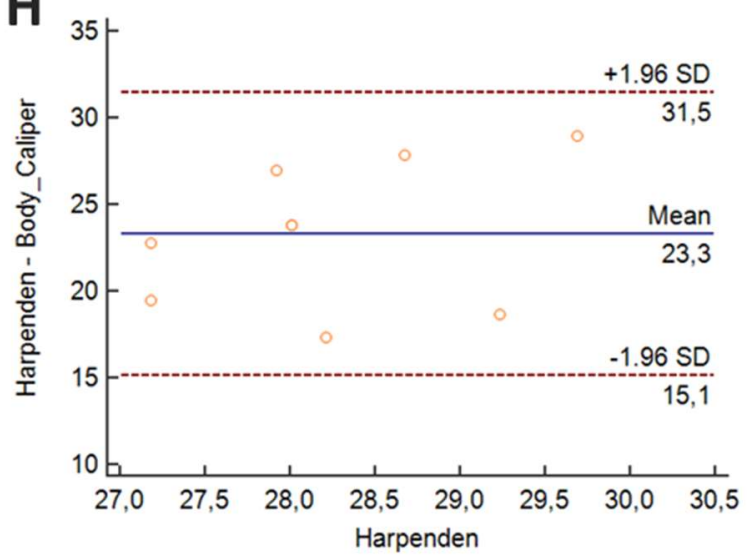

Figura 1 - Análise de Bland-Altman entre os equipamentos Harpenden e Premier (A), Digital (B), Innovare (C), Holtain (D), Lange (E), Slim Guide (F), Neo Prime (G) e Body Caliper (F). 
Os demais equipamentos não obtiveram níveis adequados de precisão e exatidão $(\mathrm{F}, \mathrm{G}$ e H).

\section{Discussão}

Os dados mostraram de forma consistente que o uso de diferentes plicômetros pode intervir na análise da composição corporal e inviabilizar a comparação de dados. Embora a precisão de todos os modelos seja satisfatória (exceto o Body Caliper), 63\% dos equipamentos avaliados não exibiram níveis aceitáveis de pressão ao serem comparados ao padrão ouro (Harpenden). Somente os modelos nacionais (Innovare, Premier e Digital) proporcionaram níveis aceitáveis de exatidão. Estes achados mostram-se relevantes por esta pesquisa ter sido a primeira a avaliar de forma abrangente a pressão realizada por diferentes plicômetros disponíveis no mercado nacional e internacional.

De acordo com Cyrino et al.(2), o modelo do plicômetro e a falta de calibração do mesmo, são fatores determinantes para obtenção de valores precisos e confiáveis. Gore et al.(10) complementam mencionando que a deterioração das molas pode afetar a acurácia da medida. Para minimizar este viés, os equipamentos utilizados eram novos e foram previamente calibrados. As linhas horizontais da Gráfico 1 evidenciam que os modelos Body Caliper, Neo Prime e Slim Guide não conseguem sustentar a pressão de forma homogênea com o incremento da amplitude. $\mathrm{O}$ posicionamento das molas e o comprimento das hastes são fatores que justificam este comportamento (Quadro 1).

Segundo Edwards et al.(7), para que um plicômetro seja preciso e suas medidas confiáveis é necessário que algumas características sejam respeitadas para manter a tensão da mola constante $\left(8-10 \mathrm{~g} / \mathrm{mm}^{2}\right)$ ao longo da abertura $(0-80 \mathrm{~mm})$. As duas principais orientações são(1): a distância das áreas de contato do pivô (152,4 mm) e o ângulo das molas para compensar a Lei de Hooke, a qual determina que a tensão nas molas seja proporcional à sua deformação, ou seja, ao comprimento ocasionado por forças externas(11,12). Se olharmos com atenção as características dos equipamentos (Tabela 1), torna-se evidente que os modelos Body Caliper, Lange, Neo Prime e Slim Guide não cumprem estes requisitos recomendados por Edwards et al. (7). Ainda assim, a Tabela 1 indica que, em média, os equipamentos $\mathrm{Neo}$ Prime e Slim Guide foram precisos.

Em relação à exatidão (Gráfico 1), é possível observar que somente os modelos Innovare, Premier e Digital demonstraram comportamento similar ao padrão de referência. Informação ratificada pelo ETMINTER (Tabela 1) e análise de confiabilidade (Figura 1). Embora os modelos Holtain e Lange tenham mantido a pressão constante com aumento da amplitude, suas pressões foram 20 e $45 \%$ menor que a medida padrão, respectivamente. Dois fatores podem explicar esta resposta: a posição vertical da única mola e a distância das áreas de contato do plicômetro Lange e, talvez, o posicionamento das molas do Holtain. Curiosamente, as amplitudes de 10, 15 e $20 \mathrm{~mm}$ exibiram maior ETM-INTER (> $20 \%$ ) no modelo inglês.

Existem poucos estudos comparando o uso de diferentes plicômetros na análise da composição corporal e, até o momento, nenhum deles investigou de forma mais incisiva a precisão e a exatidão destes equipamentos. Cyrino et al. (2) foram pioneiros ao compararem a espessura de DC usando dois plicômetros distintos (Lange e Cescorf) e o impacto que isso causava na análise da composição corporal de 259 homens. Foram aferidas nove DC com cada equipamento, em alternância (três medidas cada). A mediana foi usada para cálculo da densidade corporal, sendo posteriormente convertida em percentual de gordura $(\% \mathrm{G})$ usando equações específicas.

Os autores observaram que o modelo Lange registrava valores maiores de DC que o Cescorf ( $\Sigma 9 \mathrm{DC} 112,6 \pm 7,6$ vs 105,6 \pm 7,9 mm; $\mathrm{p}<0,01)$ e que estas medidas prediziam um $\% \mathrm{G}$ distinto nas quatro equações investigadas $(\sim 15$ $\pm 6 v s \sim 14 \pm 6 \% ; \mathrm{p}<0,01)$. Ainda que os métodos sejam distintos, estes dados corroboram com os obtidos em nosso estudo. Se observarmos a Gráfico 1 pode-se notar que a tensão gerada pelo modelo Lange é inferior a todos os modelos Cescorf, consequentemente, registrando valores menores devido a menor 
compressibilidade dos tecidos moles. Neste sentido, Gore et al.(10) afirmam que variações de $1 \mathrm{~g} / \mathrm{mm}^{2}$ podem diminuir a espessura da DC em aproximadamente 10\%, independentemente do local de medida.

Corroborando com estes achados, Gruber et al. (5) identificaram um ETM-INTRA próximo a $11 \mathrm{~mm}(\Sigma 7 \mathrm{DC})$ em homens $(104,5 \pm 36,3 v s$ $93,8 \pm 32,8 \mathrm{~mm} ; \mathrm{p}<0,05)$ e mulheres saudáveis $(113,5 \pm 39,3$ vs $103,7 \pm 37,3 \mathrm{~mm} ; \mathrm{p}<0,05)$. Assim como no estudo anterior, os valores obtidos usando o Lange foram menores que o Harpenden, ainda que altamente correlacionadas $(\mathrm{r}=0,99)$. Por outro lado, Talbert et al.(6) não observaram distinção no $\% \mathrm{G}$ usando os modelos Lange e Harpenden para coletar as medidas $(22,4 \pm 6,7 \quad v s$ $20,0 \pm 6,4 \%$; $\mathrm{p}=0,07)$. Todavia, o aparelho norte-americano mostrou menor sensibilidade para medir as DC, assim como observado no presente estudo.

Em relação ao Slim Guide, Hewitt et al.(13) fizeram uma série de constatações ao investigarem a calibração dinâmica nos plicômetros Slim Guide e Harpenden. Embora não fosse o foco do estudo, os autores citam que a qualidade mecânica no instrumento canadense é inferior à inglesa, apresentando maior variabilidade devido à simplicidade no mecanismo do pivô. Nossos dados apoiam esta afirmação ao demonstrarem que a tensão gerada por este equipamento aumentava de forma sistemática com a abertura das hastes (Gráfico 1), chegando a oscilar cerca de $67 \%$ entre as amplitudes de 10 e $45 \mathrm{~mm}$. Não encontramos estudos que tenham avaliado os modelos Neo Prime, Holtain e Body Caliper, dificultando a discussão de dados.

\section{Pontos fortes e limitações do estudo}

O ponto forte do estudo foi a comparação entre diferentes plicômetros disponíveis no mercado, evidenciando a precisão e a exatidão de cada equipamento.

Destaca-se no presente estudo a comparação entre diferentes equipamentos que são comercializados para mensurar as dobras cutâneas e como a sua variabilidade pode interferir na análise da composição corporal. Por outro lado, a ausência de testes em um número maior de equipamentos do mesmo modelo e avaliação da durabilidade dos mesmos, além da aplicabilidade em modelos humanos (coleta de medidas), constituem algumas limitações do presente estudo.

\section{Conclusão}

Atualmente diversos equipamentos são comercializados para mensurar DC e avaliar a composição corporal de atletas, enfermos e da população em geral. As opções variam desde os modelos mais simples (clínicos) até os mais avançados (científicos) e modernos (digitais). Cabe ao antropometrista optar pelo instrumento que melhor se adeque a sua realidade profissional. No entanto, é preciso ficar atento a estas diferenças mecânicas para que não ocorram erros na interpretação de dados. Basicamente, os plicômetros devem respeitar algumas premissas básicas para que os dados sejam exatos e confiáveis. Além disso, seus usuários devem inspecionar sua calibração regularmente.

Considerando os resultados deste estudo, conclui-se que há grande variabilidade entre os equipamentos desenvolvidos para mensurar as DC, podendo comprometer a confiabilidade dos dados mensurados e, consequentemente, a análise da composição corporal. Dentre os plicômetros avaliados mostramos que: A) os modelos Innovare, Premier e Digital são os mais próximos do padrão ouro; B) Body Caliper e Lange são os equipamentos que geram menor pressão, e por isso, suas medidas podem superestimar a composição corporal; C) os modelos Neo Prime e Slim Guide se diferenciam do padrão de referência por produzirem maior pressão, subestimando o valor da dobra cutânea; D) o plicômetro Holtain se mostrou extremamente preciso, porém, pouco exato. Portanto, a escolha do equipamento e o cálculo do ETM, são determinantes para obtenção de dados confiáveis que descrevam a composição corporal dos sujeitos.

\section{Declaração de conflito de interesses}

Os autores declaram não haver conflito de interesse.

\section{Declaração de financiamento}

Este estudo foi financiado em parte pela Fundação de Amparo à Pesquisa do Estado do Rio Grande do Sul (FAPERGS)/Coordenação 
de Aperfeiçoamento de Pessoal de Nível Superior (CAPES) - Código Financeiro 001.

\section{Referências}

1. Perini T, Oliveira G, Ornellas J, Oliveira F. Cálculo do erro técnico de medição em antropometria. Revista Brasileira de Medicina do Esporte. 2005;11(1):81-5. http://dx.doi.org/10.1590/S151786922005000100009

2. Cyrino E, Okano A, Glaner $\mathrm{M}$, et al. Impact of the use of different skinfold calipers for the analysis of the body composition. Revista Brasileira de Medicina do Esporte. 2003;9(3):150-3. http://dx.doi.org/10.1590/S151786922003000300004

3. Both D, Matheus S, Behenck M. Validação de equações antropométricas específicas e generalizadas para estimativa do percentual de gordura corporal em estudantes de educação física do sexo masculino. Revista Brasileira de Educação Física e Esporte. 2015;29(1):13-23.

http://dx.doi.org/10.1590/180755092015000100013

4. Lopes A, Ribeiro G. Antropometria aplicada à saúde e ao desempenho esportivo: uma abordagem a partir da metodologia ISAK. Rio de Janeiro: Rúbio; 2014.

5. Gruber J, Pollock M, Graves J, et al. Comparison of Harpenden and Lange calipers in predicting body composition. Research Quarterly for Exercise and Sport. 1990;61(2):184-90. DOI: $10.1080 / 02701367.1990 .10608673$

6. Talbert E, Flynn M, Bell J, et al. Comparison of body composition measurements using a new caliper, two established calipers, hydrostatic weighing, and BodPod. International Journal of Exercise Science. 2009;2(1):19-27.

7. Edwards D, Hammond W, Healy M, Tanner J, Whitehouse R. Design and accuracy of calipers for measuring subcutaneous tissue thickness. British Journal of Nutrition. 1955;9(2):133-43.
8. Global Market Insights. Body fat measurement market size: industry analysis report, regional outlook, growth potential, price trends and forecast 20162024. Delaware: Global Market Insight, $2016 . \quad$ Disponível em: https://www.gminsights.com/industryanalysis/body-fat-measurement-market. Acessado em: 04/07/2019.

9. Hirakata V, Camey S. Análise de Concordância entre Métodos de BlandAltman. Clinical \& Biomedical Research. 2009; 29(3): 261-68.

10. Gore C, Carlyon R, Franks S, Woolford S. Skinfold thickness varies directly with spring coefficient and inversely with jaw pressure. Medicine \& Science in Sports \& Exercise. 2000;32(2):540-6.

11. Gore C, Woolford S, Carlyon R. Calibrating skinfold calipers. Journal of Sports Sciences. 1995;13(4):355-60.

12. Carlyon R, Bryant R, Gore C, Walker R. Apparatus for precision calibration of skinfold calipers. American Journal of Human Biology. 1998;10(6):689-97.

13. Hewitt G, Withers R, Brooks A, et al. Improved rig for dynamically calibrating skinfold calipers: comparison between Harpenden and Slim Guide instruments. American Journal of Human Biology. 2002;14(6):721-727. 